\title{
Early Post-partum Glucose Intolerance, Metabolic Syndrome and Gestational Diabetes Mellitus Determinants After Assisted Conception: A Prospective Cohort Study
}

\section{Azam Kouhkan ( $\nabla$ akouhkan@royaninstitute.org)}

Department of Diabetes, Obesity and Metabolism, Reproductive Biomedicine Research Center, Royan Institute for Reproductive Biomedicine, ACECR, Tehran, Iran https://orcid.org/0000-0001-5224-577X Roya Hosseini

Royan Institute

Hamid Reza Baradaran

Iran University of Medical Sciences

Arezoo Arabipoor

Royan Institute

Rezvaneh Cheraghi

Royan Institute

Ashraf Moini

Tehran University of Medical Sciences School of Medicine

\section{Farideh Malekzadeh}

Royan Institute

Mohammad E. Khamseh

Iran University of Medical Sciences

Research article

Keywords: Early post-partum, Glucose intolerance, metabolic syndrome, Gestational diabetes mellitus, Assisted Reproduction technology

Posted Date: August 25th, 2020

DOl: https://doi.org/10.21203/rs.3.rs-61706/v1

License: (c) (i) This work is licensed under a Creative Commons Attribution 4.0 International License. Read Full License 


\section{Abstract}

Background: This study aimed to determine the prevalence of postpartum metabolic syndrome (MetS), glucose intolerance and other determinants, 6-12 weeks postpartum in women with assisted reproduction technology conception gestational diabetes mellitus diagnosis (ART-GDM) compared to women with spontaneous conception and GDM diagnosis (SC-GDM).

Methods: In this prospective cohort study, two groups consisted of 62 ART-GDM and 64 SC-GDM singleton pregnant women were followed 6-12 weeks after delivery for postpartum MetS. Fasting glucose, 75-g 2-h OGTT and lipid profile were assessed. Waist and hip circumference, and systolic and diastolic blood pressures (BP) were measured at post- partum. Clinical, para clinical and obstetric data were recorded from registry offices. The prevalence of MetS and glucose intolerance were determined. Predictors of Mets and glucose intolerance were determined by logistic regression.

Results: The prevalence of postpartum MetS was $20.8 \%$ in ART-GDM women and $10.9 \%$ in SC-GDM, $\mathrm{P}=0.123)$. Mean postpartum BMI and systolic BP were significantly higher in the ART-GDM group $(P=0.016$ and $P=0.027$, respectively). Adverse pregnancy outcomes were significantly higher in the ARTGDM group. Postpartum glucose intolerance prevalence did not vary significantly between the groups. Family history of diabetes was a predictive factor for postpartum MetS and glucose intolerance 6-12 weeks after delivery.

Conclusions: Early postpartum MetS and glucose intolerance prevalence after assisted conception did not vary significantly; however, postpartum BMI and systolic BP were significantly higher in the ART-GDM group. Lifestyle modification program and long-term health care of ART women with GDM diagnosis can be recommended. Further studies with larger sample size and longer follow-up are necessary to verify our findings.

\section{Background}

Gestational diabetes mellitus (GDM) recognized as any degree of glucose intolerance with onset or first recognition during the second or third trimester of pregnancy, is one of the most prevalent metabolic disorders[1]. The apparent rise of GDM incidence could be due to advanced maternal age in pregnancy, application of the new diagnostic criteria with lower threshold and single abnormal value, or increasing trend of obesity and unhealthy diet in the general population $[2,3]$.

The metabolic perimeter of GDM in young women may persist in type $2 \mathrm{DM}$ and in the form of metabolic syndrome (MetS) [4]. There are some conflicting evidence on the relationship between GDM and early increased occurrence of MetS [4-6]; it is likely that GDM contributes to early expression of MetS in some mothers and their child $[4,6,7]$. MetS and GDM have common similar clinical features [8] and the prevalence of MteS shows 3- to 4- fold increases in women with a history of GDM[9]. Previous data showed that MetS features such as glucose intolerance, low high density lipoprotein cholesterol (HDL-C), hypertriglyceridemia, hypertension, and central obesity, are commonly observed in women post GDM [10]. 
These conditions could be associated with hypertension, dyslipidemia and cardiovascular disease [11, 12]. Considering the increased risk of the above-mentioned complications in GDM and MetS and their interconnected nature, it is worth identifying risk factors of early postpartum MetS in women with GDM diagnosis.

Moreover, recent evidence suggests that the risk of GDM and the need for insulin therapy during pregnancy increases in pregnancy induced by assisted reproductive technology (ART) $[13,14]$. Some mechanisms that might explain this increased risk in infertile population include higher rates of advanced maternal age and obesity, infertility etiology as well as the treatment procedures, drugs, and epigenetic modifications $[15,16]$. As a first study, it would be interesting to investigate whether the risk of early postpartum MetS increases in this population.

Based on the importance of the topic, this study was designed to determine the relationship between GDM and early postpartum MetS. The primary aims were to compare the prevalence of MetS and glucose intolerance at 6-12 weeks postpartum, between spontaneous and ART pregnancies. The secondary aims were to determine the contributing risk factors in both populations.

\section{Methods}

\section{Study design and settings}

In this prospective cohort study, the ART and spontaneous pregnancies populations including all singleton pregnant women diagnosed with GDM at 24-28 weeks of pregnancy, were followed at Royan Institute (Endocrinology and Female Infertility Clinic) and Arash women's Hospital (maternity teaching hospital in Tehran) respectively, between 2015 and 2017. This research project was approved by the institutional review board and Ethics Committee of Iran University of Medical Sciences, Iran and Royan Institute, Iran. All participants signed an informed consent after ensuring confidentiality and that the data will be reported anonymously. The inclusion criteria were having GDM the in second or third trimester confirmed by 75-g OGTT (one-step glucose tolerance test) and availability of clinical and medical records.

Women of $\geq 42$ years old, with pre-gestational diabetes type 1 or 2 , multiple pregnancy and chronic diseases such as hypertension, cardiovascular diseases, untreated thyroid disease, liver diseases, renal diseases, autoimmune diseases, and connective tissue disorders as well as those who were taking corticosteroids were excluded.

Two groups of GDM mothers (SC-GDM and ART-GDM) were followed during pregnancy, delivery and 612 weeks postpartum for assessment of adverse maternal, fetal and neonatal outcome; in addition, occurrence of MetS and glucose intolerance at postpartum was assessed.

\section{Biochemical and clinical assessment during pregnancy}

To distinguish pre-gestational diabetes, all pregnant women were evaluated during the first trimester by determination of fasting blood sugar (FBS) and the results were recorded in hospital registry office. 
Gestational diabetes was approved by OGTT using $75 \mathrm{~g}$ oral glucose at 24-28 weeks of gestation, based on ADA/ IAPDSG (American Diabetes Association/International Association of the Diabetes and Pregnancy Study Groups) criteria [1].

After 8-12-hour fasting, blood samples were collected from all patients at the second and third trimester of pregnancies. Fasting blood sugar (FBS 2nd trimester), insulin (insulin 2nd trimester), hemoglobin A1C ( $\mathrm{HbA} 1 \mathrm{c}$ 2nd trimester), and lipid profile were measured in the 2nd trimester of pregnancy. Homeostasis model assessment-insulin resistance (HOMA-IR 2nd trimester) was calculated based on the formula. Fasting blood sugar (FBS 3rd trimester (and hemoglobin A1C (HbA1c 3rd trimester) were rechecked in the 3rd trimester of pregnancy.

\section{Data Collection}

A trained physician recorded socio-demographic characteristics, medical and obstetric history, potential risk factors of GDM and MetS, and details of GDM management and delivery, as well as maternal and neonatal outcomes; also, postpartum questionnaires were completed at 6-12 weeks postpartum. Clinical data were collected from hospital records.

\section{Postpartum biochemical and clinical assessment}

Postpartum fasting blood sugar (PP FBS) determination, 75-g 2-h OGTT (PP GTT2h) and lipid profile tests (in terms of PP cholesterol, PP triglycerides, PP LDL-cholesterol, PP HDL-cholesterol and PP VLDLcholesterol) were performed at 6-12 weeks after delivery. Postpartum pre-diabetes [impaired fasting glucose (IFG) and impaired glucose tolerance (IGT)], and diabetes were defined according to the ADA criteria [17]. Weight (PP weight), height (PP height), waist circumference (PP waist) and blood pressure (PP systolic and diastolic BP) were measured at 6-12 weeks postpartum. Blood pressure was checked two times with a 30 min interval. The height and weight of each subject measured while wearing light clothing and no shoes were used to calculate body mass index (BMI). The MetS was defined by two criteria [National Cholesterol Education Program Adult Treatment Panel (NCEP ATP III) and International Diabetes Federation (IDF)]. According to NCEP ATP III criteria, MetS was diagnosed if any three of five of the following disorders was observed: waist circumference $\geq 88 \mathrm{~cm}$, triglycerides $\geq 150 \mathrm{mg} / \mathrm{dl}$, HDLcholesterol $<50 \mathrm{mg} / \mathrm{dl}, \mathrm{FPG} \geq 100 \mathrm{mg} / \mathrm{dl}$, or blood pressure $\geq 130 / 85 \mathrm{mmHg}$ [18]. The MetS was recognized based on IDF definition: central obesity (waist circumference $>88 \mathrm{~cm}$ ) plus any two of the four above-noted factors[19].

\section{Statistical analysis}

Primary aim was comparing early postpartum MetS and glucose intolerance prevalence between the study groups and secondary aim was evaluation of potential risk factors of MetS and glucose intolerance in the study population. Data were analyzed by the Stata software (Version 13.0) (STATA Corp, College Station, Texas). A p-value $<0.05$ was considered statistically significant. The normal quantity variables are presented as mean \pm standard deviation (SD). Chi- square test was applied for making comparison with respect to the categorical variables between the groups. Student's $t$ test and Mann-Whitney test were 
applied when appropriate. The multivariable logistic regression analysis was used to adjust for women's age and BMI for detection of predictive variables for early postpartum MetS and glucose intolerance in whole study population $(n=126)$. The covariate variables were FBS $2 n d$ trimester, FBS 3rd trimester, $\mathrm{HbA1c}$ 3rd trimester, family history of DM, and prior GDM that were considered in regression model.

The estimation was made by using the NCSS software (Number Cruncher Statistical System software package 2007, Kaysville, UT, USA); a sample size of 65 subjects in each group would support us to evaluate early postpartum MetS prevalence in each group with a power of $80 \%$ and a type I error of 0.05 (total sample size $=130$ ).

\section{Results}

In this study, 126 women with GDM pregnancy including 62 ART (ART-GDM) and 64 spontaneous conceptions (SC-GDM) were followed for the incidences of postpartum glucose intolerance and MetS at 6-12 weeks after delivery.

Table 1 summarizes the baseline characteristics of the women who participated in this study. There were no significant differences in mean maternal age, pre-pregnancy BMI, or systolic and diastolic blood pressure between the two groups $(P>0.05)$. In addition, the rates of first degree family history of diabetes mellitus and hypertension, as well as most history of adverse outcomes in prior pregnancy, were not significantly different between the two groups. However, the rates of nulliparity, and prior history of macrocosmic infant were significantly different between the two groups. Importantly, the results of biochemical assessments showed that mean second trimester insulin and HOMA-IR as well as third trimester FBS were significantly higher in the ART-GDM group compared to the SC-GDM group. 
Table 1

Comparison of clinical and biochemical characteristics before and during pregnancy between SC-GDM and ART-GDM groups referred for postpartum examination.

\begin{tabular}{|c|c|c|c|}
\hline Variables & $\begin{array}{l}\text { ART-GDM + } \\
(n=62)\end{array}$ & $\begin{array}{l}\text { SC-GDM+ } \\
(n=64)\end{array}$ & P-value \\
\hline \multicolumn{4}{|l|}{ Clinical } \\
\hline Maternal age (years) & $31.2 \pm 4.9$ & $32.3 \pm 4.9$ & 0.234 \\
\hline Parity $(=0)$ & $54(87.1)$ & 25(39.1) & 0.001 \\
\hline Pre-pregnancy BMI (kg/m²) & $27.3 \pm 3.8$ & $26.0 \pm 4.7$ & 0.112 \\
\hline Family history of DM & $31(50)$ & $24(37.5)$ & 0.157 \\
\hline Family history of HTN & $29(46.8)$ & $28(43.8)$ & 0.733 \\
\hline Systolic blood pressure, (mmHg) & $107.9 \pm 11.4$ & $106.7 \pm 10.5$ & 0.544 \\
\hline Diastolic blood pressure, $(\mathrm{mmHg})$ & $66.9 \pm 7.8$ & $68.4 \pm 8.6$ & 0.334 \\
\hline Prior history of GDM & $3(4.8)$ & $9(14.1)$ & 0.078 \\
\hline Prior history of pre-eclampsia, & $2(3.2)$ & $1(1.6)$ & 0.540 \\
\hline Prior history of LBW, & $1(1.6)$ & $6(9.4)$ & 0.057 \\
\hline Prior history of preterm birth, & $2(3.2)$ & $5(7.8)$ & 0.261 \\
\hline Prior history of macrosomic infant, & $0(0)$ & $5(7.8)$ & 0.025 \\
\hline Prior history of multiple pregnancies & $3(4.8)$ & $4(6.3)$ & 0.730 \\
\hline Prior history of abortion, & $42(67.7)$ & $47(74.6)$ & 0.397 \\
\hline Prior history of neonatal death & $2(3.2)$ & $1(1.56)$ & 0.540 \\
\hline Prior history of oligohydramnious & $0(0)$ & $2(3.1)$ & 0.161 \\
\hline \multicolumn{4}{|l|}{ Biochemical } \\
\hline FBS 2nd trimester, (mg/dl) & $87.9 \pm 8.4$ & $84.2 \pm 12.2$ & 0.053 \\
\hline HbA1c 2nd trimester, (mg/ dl) & $5.0 \pm 0.6$ & $4.9 \pm 0.6$ & 0.296 \\
\hline TG 2nd trimester, (mg/dl) & $191.4 \pm 60.9$ & $202.6 \pm 54.0$ & 0.294 \\
\hline Cholesterol 2nd trimester, (mg/dl) & $204.6 \pm 39.3$ & $225.4 \pm 40.0$ & 0.005 \\
\hline HDL 2nd trimester, (mg/dl) & $63.6 \pm 13.2$ & $65.8 \pm 12.8$ & 0.367 \\
\hline LDL 2nd trimester, (mg/dl) & $103.1 \pm 35.4$ & $119.1 \pm 32.8$ & 0.012 \\
\hline VLDL 2nd trimester, (mg/dl) & $37.8 \pm 10.8$ & $40.5 \pm 10.8$ & 0.177 \\
\hline
\end{tabular}




\begin{tabular}{|c|c|c|c|}
\hline Variables & $\begin{array}{l}\text { ART-GDM + } \\
(n=62)\end{array}$ & $\begin{array}{l}\text { SC-GDM+ } \\
(n=64)\end{array}$ & P-value \\
\hline Insulin 2nd trimester, (mg/dl) & $14.4 \pm 9.1$ & $11.3 \pm 5.7$ & 0.041 \\
\hline HOMA-IR 2nd trimester & $3.2 \pm 2.1$ & $2.4 \pm 1.4$ & 0.027 \\
\hline FBS 3rd trimester, (mg/dl) & $88.5 \pm 9.5$ & $83.1 \pm 10.8$ & 0.008 \\
\hline HbA1c 3rd trimester, (mg/dl) & $5.1 \pm 0.6$ & $5.0 \pm 0.7$ & 0.863 \\
\hline \multicolumn{4}{|c|}{$\begin{array}{l}\text { ART: assisted reproductive technology; GDM: gestational diabetes mellitus; SC: spontaneous } \\
\text { conception; HTN: hypertension; HDL: High-density lipoprotein; TG; Triglyceride; LDL: low-density } \\
\text { lipoprotein; VLDL: Very-low-density lipoprotein; HOMA-IR: Homeostatic Model Assessment of Insulin } \\
\text { Resistance; FBS: fasting blood sugar; HbA }{ }_{1} \text { C: Hemoglobin A1c }\end{array}$} \\
\hline
\end{tabular}

The comparison of maternal, fetal and neonatal outcomes between the SC-GDM and ART-GDM groups is presented in Table 2. Mean neonatal weight and gestational age at delivery were significantly lower in the ART-GDM group. Maternal outcomes showed that the rates of pregnancy induced hypertension (PIH) and preeclampsia were significantly higher in the ART-GDM group. In addition, the incidence of fetal and neonatal complications in terms of preterm birth, small for gestational age (SGA), neonatal intensive care unit (NICU) admission, and neonatal hypoglycemia, was significantly higher in the ART-GDM group. Other adverse pregnancy outcomes did not show any significant difference between the two groups. 
Table 2

Comparison of maternal, fetal and neonatal outcomes between SC-GDM and ART-GDM groups.

\begin{tabular}{|c|c|c|c|}
\hline Variables & $\begin{array}{l}\text { ART-GDM + } \\
(n=62)\end{array}$ & $\begin{array}{l}\text { SC-GDM+ } \\
(n=64)\end{array}$ & P-value \\
\hline Neonatal sex, Male & $25(40.3)$ & $36(56.3)$ & 0.074 \\
\hline Neonatal height, (Cm) & $49.3 \pm 3.0$ & $50.1 \pm 1.5$ & 0.084 \\
\hline Neonatal weight, $(\mathrm{Kg})$ & $3096.9 \pm 516.8$ & $3339.4 \pm 354.7$ & 0.003 \\
\hline Neonatal head circumference, $(\mathrm{Cm})$ & $34.6 \pm 1.7$ & $35.0 \pm 1.3$ & 0.121 \\
\hline Neonatal chest, $(\mathrm{Cm})$ & $32.9 \pm 2.0$ & $33.5 \pm 1.3$ & 0.119 \\
\hline Gestational age at delivery, (Wks) & $37.8 \pm 0.2$ & $38.5 \pm 0.1$ & 0.003 \\
\hline Gestational weight gain & $11.3 \pm 4.9$ & $11.5 \pm 5.6$ & 0.825 \\
\hline Delivery BMI, $\left(\mathrm{Kg} / \mathrm{m}^{2}\right)$ & $31.7 \pm 4.1$ & $30.5 \pm 4.4$ & 0.126 \\
\hline \multicolumn{4}{|l|}{ Maternal outcomes } \\
\hline $\mathrm{PIH}$ & 10(16.1) & $2(3.1)$ & 0.013 \\
\hline Preeclampsia, & $7(11.3)$ & $1(1.6)$ & 0.031 \\
\hline Antepartum hemorrhage & $9(14.5)$ & $3(4.7)$ & 0.060 \\
\hline Emergency cesarean & $25(40.3)$ & 16(25.0) & 0.066 \\
\hline PROM & $6(9.7)$ & $1(1.6)$ & 0.060 \\
\hline Oligohydramnious & $6(9.7)$ & $2(3.1)$ & 0.132 \\
\hline Polyhydramnious & $3(4.8)$ & $3(4.7)$ & 0.968 \\
\hline Fetal death & $1(1.6)$ & $0(0)$ & 0.308 \\
\hline \multicolumn{4}{|l|}{ Fetal and neonatal outcomes } \\
\hline Preterm birth & $9(14.5)$ & $1(1.6)$ & 0.008 \\
\hline IUGR & $6(9.7)$ & $2(3.1)$ & 0.125 \\
\hline SGA & $9(14.5)$ & $1(1.6)$ & 0.008 \\
\hline LGA & $2(3.2)$ & $5(7.8)$ & 0.261 \\
\hline Macrosomia & $1(1.6)$ & $4(6.3)$ & 0.189 \\
\hline \multicolumn{4}{|l|}{ LBW } \\
\hline NICU admission & 12(19.7) & $3(4.7)$ & 0.010 \\
\hline Respiratory distress & $7(11.5)$ & $4(6.3)$ & 0.303 \\
\hline
\end{tabular}




\begin{tabular}{|c|c|c|c|}
\hline Variables & $\begin{array}{l}\text { ART-GDM + } \\
(n=62)\end{array}$ & $\begin{array}{l}\text { SC-GDM+ } \\
(n=64)\end{array}$ & P-value \\
\hline Neonatal hypoglycemia & $8(13.1)$ & $2(3.1)$ & 0.040 \\
\hline Perinatal mortality & $1(1.6)$ & $0(0)$ & 0.308 \\
\hline Apgar $<7$ at $5 \mathrm{~min}$ & $2(3.2)$ & $1(1.6)$ & 0.559 \\
\hline Birth trauma & $0(0)$ & $2(3.1)$ & 0.496 \\
\hline \multicolumn{4}{|c|}{$\begin{array}{l}\text { The quantitative and qualitative variables are presented as Mean } \pm \text { SD and } n(\%) \text {, respectively } \\
\text { ART: assisted reproductive technology; BMI: body mass index; GDM: gestational diabetes mellitus; } \\
\text { SC: spontaneous conception; PROM: Premature rupture of membranes; IUGR: Intrauterine growth } \\
\text { restriction; SGA: Small for gestational age; LGA: large for gestational age; LBW: Low birth weight; } \\
\text { NICU: neonatal intensive care unit }\end{array}$} \\
\hline
\end{tabular}

Table 3 compares the clinical and laboratory characteristics within 6-12 weeks after delivery between the SC-GDM and ART-GDM groups. The results of postpartum metabolic parameters revealed no significant differences between the two groups except for mean BMI and systolic blood pressure which were higher in the ART-GDM group. The parameters included in the diagnostic criteria of MetS-fasting plasma glucose, waist circumference, triglyceride, and HDL cholesterol were not significantly different between the ART-GDM and SC-GDM groups except for systolic blood pressure. Additionally, the 2-h glucose after 75-g GTT was not significantly different between the ART-GDM and SC-GDM women. The frequency of MetS using the NCEP ATP III criterion was 10.9 and $20.8 \%$ in the SC-GDM and ART-GDM group, respectively. The univariate analysis presented that the odds ratio of postpartum MetS using NCEP ATP III Criteria, did not vary significantly between ART and spontaneous GDM pregnancies after adjustment for age, and BMI [aOR; 1.88(0.68-5.22)]. These values for MetS obtained through the IDF criterion were 17.2 and 19.4, respectively. However, the rate of postpartum glucose abnormalities including pre-diabetes [impaired fasting glucose (IFG) and impaired glucose tolerance (IGT)], and diabetes did not show any significant differences between the two groups. 
Table 3

Comparison of postpartum parameters between SC-GDM and ART-GDM groups.

\begin{tabular}{|c|c|c|c|}
\hline Variables & $\begin{array}{l}\text { ART-GDM + } \\
(n=62)\end{array}$ & $\begin{array}{l}\text { SC-GDM+ } \\
(n=64)\end{array}$ & P-value \\
\hline PP weight, (Kg) & $74.2 \pm 13.1$ & $69.6 \pm 12.7$ & 0.052 \\
\hline PP BMI, $\left(\mathrm{Kg} / \mathrm{m}^{2}\right)$ & $28.8 \pm 4.4$ & $26.7 \pm 4.5$ & 0.016 \\
\hline PP waist, (Cm) & $92.6 \pm 9.8$ & $92.2 \pm 10.3$ & 0.734 \\
\hline PP Hip,(Cm) & $107.9 \pm 1.3$ & $105.5 \pm 1.1$ & 0.167 \\
\hline PP Systolic BP,(mmHg) & $110.5 \pm 10.2$ & $104.9 \pm 16.3$ & 0.027 \\
\hline PP Diastolic BP,(mmHg) & $69.5 \pm 8.1$ & $68.9 \pm 8.3$ & 0.683 \\
\hline PP FBS, (mg/ dl) & $94.6 \pm 10.7$ & $92.2 \pm 13.4$ & 0.261 \\
\hline PP GTT2h, (mg/ dl) & $104.4 \pm 3.9$ & $105.0 \pm 3.2$ & 0.902 \\
\hline PP TG, (mg/ dl) & $114.2 \pm 91.9$ & $116.6 \pm 58.2$ & 0.827 \\
\hline PP cholesterol, (mg/ dl) & $178.7 \pm 31.4$ & $186.7 \pm 35.8$ & 0.185 \\
\hline PP HDL, (mg/ dl) & $55.1 \pm 11.5$ & $55.3 \pm 10.8$ & 0.946 \\
\hline PP LDL, (mg/ dl & $100.9 \pm 27.8$ & $107.5 \pm 26.8$ & 0.179 \\
\hline PP VLDL, (mg/ dl) & $21.6 \pm 11.9$ & $22.6 \pm 10.9$ & 0.657 \\
\hline PP metabolic syndrome & $13(20.8)$ & $7(10.9)$ & 0.123 \\
\hline PP GTT 75 gr result, & $45(73.8)$ & $51(79.7)$ & 0.718 \\
\hline Normal FBS & $14(23.0)$ & $11(17.2)$ & \\
\hline Pre-diabetes (IGT or IFG) & $2(3.3)$ & $2(3.1)$ & \\
\hline \multicolumn{4}{|l|}{ DM } \\
\hline PP GTT 75 gr result & $45(73.8)$ & $51(79.7)$ & 0.433 \\
\hline Normal FBS & $16(26.2)$ & $13(20.3)$ & \\
\hline \multicolumn{4}{|c|}{ Glucose intolerance (pre-diabetes + DM) } \\
\hline MetS using NCEP ATP III criteria & $13(20.8)$ & $7(10.9)$ & 0.123 \\
\hline MetS using IDF Criteria & 12(19.4) & 11(17.2) & 0.753 \\
\hline
\end{tabular}


Table 4

Association of clinical and biochemical parameters and postpartum glucose intolerance and metabolic syndrome in GDM population

\begin{tabular}{|c|c|c|c|c|}
\hline \multirow[t]{2}{*}{ Variables } & \multicolumn{2}{|c|}{$\begin{array}{l}\text { PP glucose intolerance } \\
\text { (pre-diabetes/diabetes) }\end{array}$} & \multicolumn{2}{|c|}{ PP metabolic syndrome } \\
\hline & OR, Cl 95\% & P-Value & OR, Cl 95\% & P-Value \\
\hline Age & $1.03(0.95-1.12)$ & 0.462 & $1.0(0.9-1.1)$ & 0.871 \\
\hline Pre-pregnancy BMI,(Kg/m²) & $1.04(0.94-1.14)$ & 0.448 & $1.20(1.1-1.32)$ & 0.004 \\
\hline 2nd trimester Systolic BP,(mmHg) & $1.00(0.97-1.04)$ & 0.839 & $1.0(0.9-1.1)$ & 0.174 \\
\hline 2nd trimester Diastolic BP,(mmHg) & $1.03(0.98-1.09)$ & 0.187 & $1.0(0.9-1.1)$ & 0.696 \\
\hline 2nd trimester FBS, (mg/ dl) & $1.06(1.01-1.10)$ & 0.009 & $1.06(1.01-1.11)$ & 0.011 \\
\hline 2nd trimester $\mathrm{HbA1c}$, (mg/ dl) & $1.34(0.69-2.57)$ & 0.387 & $1.30(0.6-2.8)$ & 0.535 \\
\hline 2nd trimester TG, (mg/ dl) & $1.00(0.99-1.01)$ & 0.781 & $1.01(1.001-1.02)$ & 0.016 \\
\hline 2nd trimester Cholesterol, (mg/ dl) & $0.99(0.98-1.01)$ & 0.097 & $1.0(0.9-1.0)$ & 0.167 \\
\hline 2nd trimester HDL, (mg/ dl) & $0.98(0.94-1.01)$ & 0.213 & $0.96(0.92-0.99)$ & 0.040 \\
\hline 2nd trimester LDL, (mg/ dl) & $0.99(0.97-1.00)$ & 0.117 & $0.9(0.8-1.1)$ & 0.122 \\
\hline 2nd trimester VLDL, (mg/ dl) & $1.00(0.97-1.05)$ & 0.662 & $1.04(1.0-1.1)$ & 0.074 \\
\hline 2nd trimester Insulin, (mg/ dl) & $1.06(0.99-1.15)$ & 0.083 & $1.07(1.01-1.14)$ & 0.040 \\
\hline 2nd trimester HOMA-IR & $1.25(0.96-1.62)$ & 0.091 & $1.40(1.02-1.78)$ & 0.034 \\
\hline 3rd trimester FBS, (mg/ dl) & $1.10(1.04-1.16)$ & 0.001 & $1.04(0.99-1.10)$ & 0.105 \\
\hline 3rd trimester $\mathrm{HbA} 1 \mathrm{c},(\mathrm{mg} / \mathrm{dl})$ & $3.04(1.02-7.65)$ & 0.019 & $2.21(0.81-5.94)$ & 0.118 \\
\hline Family history of DM & $2.54(1.07-6.01)$ & 0.034 & $2.19(0.83-5.83)$ & 0.113 \\
\hline Gravida & $1.17(0.48-2.80)$ & 0.733 & $0.80(0.33-2.41)$ & 0.817 \\
\hline Parity & $1.68(0.61-4.61)$ & 0.313 & $0.89(0.20-2.22)$ & 0.517 \\
\hline Prior GDM & $4.60(1.29-16.33)$ & 0.018 & $0.40(0.09-1.75)$ & 0.223 \\
\hline
\end{tabular}

Multivariable logistic regression presented potential risk factors for MetS and glucose intolerance, 6-12 weeks postpartum (Table 5). The results showed family history of diabetes (OR $=3.37 ; 95 \% \mathrm{Cl}$ : $1.10-$ $10.30 ; P=0.033$ ) as a predictive factor for early postpartum MetS. In addition, family history of diabetes 
$(\mathrm{OR}=2.69 ; 95 \% \mathrm{Cl}: 1.17-6.15 ; \mathrm{P}=0.019)$ and second trimester FBS $(\mathrm{OR}=1.06 ; 95 \% \mathrm{Cl}: 1.02-1.11 ; \mathrm{P}=$ 0.004 ) were independent predictors of glucose intolerance, $6-12$ weeks after delivery.

Table 5

Multivariable logistic regression for detection of risk factors of early postpartum metabolic syndrome and glucose intolerance in the study population.

\begin{tabular}{|lllll|}
\hline Variables & $\begin{array}{l}\text { Model 1 For PP metabolic } \\
\text { syndrome } \\
\text { OR }(95 \% \mathrm{Cl})\end{array}$ & $\begin{array}{l}\text { P } \\
\text { value }\end{array}$ & $\begin{array}{l}\text { Model 2 For PP } \\
\text { Glucose } \\
\text { intoleranse } \\
\text { OR (95\% Cl) }\end{array}$ & $\begin{array}{l}\text { P } \\
\text { value }\end{array}$ \\
\hline $\begin{array}{l}\text { Family history of } \\
\text { DM }\end{array}$ & $3.37(1.10-10.30)$ & 0.033 & $2.69(1.17-6.15)$ & 0.019 \\
\hline Prior GDM & $2.01(0.40-9.98)$ & 0.393 & $1.78(0.52-6.15)$ & 0.363 \\
\hline 2nd trimester FBS & 1.03 (0.97-1.09) & 0.354 & $1.06(1.02-1.11)$ & 0.004 \\
\hline $\begin{array}{l}\text { GDM: gestational diabetes mellitus; PP: postpartum; OR: odds ratio; Cl: confidence interval; FBS: } \\
\text { fasting blood sugar; DM: Diabetes Mellitus }\end{array}$ & & & \\
\hline
\end{tabular}

\section{Discussion}

Metabolic syndrome (MteS) is comprised of a cluster of glucose intolerance, hypertension, and dyslipidemia with abdominal adiposity. It is a well-known predisposing factor for insulin resistance, diabetes mellitus, and cardiovascular diseases and is rising rapidly worldwide particularly in Western and Asian countries [10]. A recent meta-analysis reported increased (approximately 4-fold) risk of MteS after GDM especially in Caucasian and obese mothers[20].

The present study compared the delivery and postpartum outcomes of GDM between ART and spontaneous pregnancies, with regard to early MteS and glucose intolerance and their components. Our results showed a higher prevalence of MteS according to the NCEP criteria in the ART-GDM (20.8\%) compared to the SC-GDM (10.9\%) group. The prevalence of MteS was 19.4\% in the GDM-ART and 17.2\% in the SC-GDM based on the IDF criteria. Moreover, there was an $88 \%$ increase in the risk of developing MteS following ART pregnancy; nevertheless, in our study, the overall differences between the two groups were not statistically significant. Numerous studies have demonstrated higher rates of postpartum MteS in GDM women compared to control group within 1-11 years postpartum, with large variations according to the length of follow- up (7.5-60\% in GDM and 4.6-26\% in non-GDM women)[2]. However, few investigations have reported MteS at 6-12 weeks after gestational diabetes and there is no evidence in GDM following assisted conception. Recently, Nouhjah et al. observed that the frequency of early postpartum MteS was $18.2 \%$ in women with GDM and $11.6 \%$ in controls by the NCEP criteria, and $21 \%$ in women with gestational diabetes and $15.1 \%$ in controls by the IDF criteria [2]. 
Current findings showed that the incidence of postpartum glucose abnormalities included pre-diabetes ( 23 vs. $17.2 \%$ ) and diabetes (3.3 vs. $3.1 \%$ ) was not significantly different between GDM after ART and spontaneous conception. In a recent meta-analysis, the prevalence of pre-diabetes and diabetes was respectively $3.9-50.9 \%$ and $2.8-58 \%$ in Asian women with gestational diabetes within 4 weeks to 15 years postpartum based on the length of follow-up [21]. Considerable evidences propose that beta-cell dysfunction likely contributes to increase the risk of glucose intolerance in the first year postpartum in GDM women [22-24].

On the basis of our data, in univariate analyses, FBS, HbA1c, family history of diabetes in first relatives, and prior GDM were risk factors for postpartum glucose intolerance in GDM population. Furthermore, prepregnancy BMI, and second trimester levels of FBS, insulin, HDL and insulin resistance, were risk factors for postpartum MteS in GDM population. Multivariate analyses confirmed family history of diabetes as an independent predictor of both glucose intolerance and MteS 6 to 12 weeks postpartum, in GDM population. Additionally, second trimester FBS was another predictive factor of glucose intolerance 6-12 weeks after delivery. Numerous researches indicate several putative factors for postpartum glucose intolerance including family history of diabetes, elevated glucose level $120 \mathrm{~min}$ after a 75-g OGTT, elevated $\mathrm{HbA}_{1} \mathrm{c}$ levels during GDM diagnosis, perinatal complications, history of GDM, obesity, systolic or diastolic blood pressure, maternal age, parity, and insulin or metformin therapy [25-29]. Additionally, history of GDM, pre-pregnancy overweight or obesity, pregnancy systolic blood pressure, or requiring insulin or metformin were reported as predisposing factors that predict postpartum MteS in GDM population $[2,20]$.

Though, there were no significant differences in baseline characteristics such as mean maternal age, prepregnancy BMI, systolic and diastolic BP between the two groups, interestingly, our findings demonstrated that mean postpartum BMI and systolic blood pressure were significantly higher in the ARTGDM group. In addition, higher second trimester insulin and insulin resistance

Several investigations have reported increased blood pressure, dyslipidemia, and higher fasting glucose levels in ART-conceived children [30-32]. The current study showed the impact of mode of conception on delivery and postpartum outcomes of GDM pregnancies especially in mothers. The pathophysiological mechanisms underlying the MteS are on debate, but insulin resistance and visceral obesity are considered major causes. The presence of at least three of five criteria of MteS is link to an increased risk of heart disease, stroke, and diabetes.

However, it is not clear that whether early postpartum raised BMI and BP on future MteS in ART mothers. Moreover, ART mothers were suffering from anxiety and stress, hormonal and environmental alternations, ex vivo manipulations, inflammatory changes, endothelial dysfunction, metabolic disturbance and medical procedures during their pregnancies $[33,34]$. These conditions may influence long-term women's health and predispose occurrence of MteS and its components. 
By the way, our study has several limitations needed to be addressed. First, this study lacks information regarding subfertility and infertility treatments during pregnancy. Second, we did not evaluate postpartum MteS and glucose intolerance in general population. Further prospective studies with larger sample size, particularly with inclusion a new group (natural pregnancy without GDM) and long-term follow up are required to verify our results.

\section{Conclusion}

In summary, the present study indicated a higher rate of MteS in ART women with GDM at 6-12 weeks postpartum compared to SC women with GDM; however, the difference was not statistically significant. Postpartum BMI and systolic BP were significantly higher in the ART-GDM group. Further investigations with larger sample-size and longer follow-up are necessary to verify our findings. Lifestyle modification and long-term health care of ART women with GDM can be recommended.

\section{Abbreviations}

MetS

postpartum metabolic syndrome; ART:assisted reproduction technology; GDM:gestational diabetes mellitus diagnosis; SC:spontaneous conception; OGTT:one-step glucose tolerance test; HOMAIR:Homeostasis model assessment-insulin resistance; FBS:fasting blood sugar; HbA1c:hemoglobin A1C; IFG:impaired fasting glucose; IGT:impaired glucose tolerance; IDF:International Diabetes Federation; BMI:body mass index; PIH:pregnancy induced hypertension; SGA:gestational age; NICU:neonatal intensive care unit; SD:standard deviation; aOR:adjusted odds ratio; Cl:confidence interval.

\section{Declarations}

\section{Ethics approval and consent to participate}

All study procedures were performed in accordance with the ethical standards of Iran University of Medical Sciences and Royan Institute ethics committees and the 1964 Helsinki declaration and its later amendments or comparable ethical standards. Ethics approval was received (date: 2015-09-4, approval No. IR.IUMS.REC.1396.25469 and date: 2014-09-4, approval No. IR.ACECR.ROYAN.REC.1393.2). Informed written consent was obtained from all individual participants included in the study

\section{Consent for publication}

Not applicable.

\section{Availability of data and materials}

The datasets used and/or analyzed during the current study are available from the corresponding author on reasonable request. 
Competing interests

The authors declare that they have no competing interests.

\section{Funding}

No financial support was received.

\section{Authors' contributions}

AK and AM: the conception, AK, HR-B and ME-Kh: design of the work; AK, RH, AA and R-Ch: the acquisition and analysis, AK, AA and FM interpretation of data; $A K, A A$ and $R-C h$ have drafted the manuscript and revised it. All authors have read and approved the manuscript.

\section{Acknowledgements}

Not applicable.

\section{Acknowledgments}

The authors would like to thank Iran universities and hospitals related to Ministry of Health and Medical Education and colleagues in Royan institute for their invaluable contributions to this research.

\section{References}

1. Association AD. (2019) 2. Classification and Diagnosis of Diabetes: Standards of Medical Care in Diabetes-2019. Diabetes care;42(Suppl 1):S13.

2. Nouhjah S, Shahbazian H, Shahbazian N, et al. (2018) Early postpartum metabolic syndrome in women with or without gestational diabetes: Results from Life after Gestational Diabetes Ahvaz cohort study. Diabetes \& Metabolic Syndrome: Clinical Research \& Reviews;12(3):317 - 23.

3. Anna V, Van Der Ploeg HP, Cheung NW, Huxley RR, Bauman AE. (2008) Sociodemographic correlates of the increasing trend in prevalence of gestational diabetes mellitus in a large population of women between 1995 and 2005. Diabetes care;31(12):2288-93.

4. Barquiel B, Herranz L, Hillman N, Burgos M, Pallardo LF. (2014) Prepregnancy body mass index and prenatal fasting glucose are effective predictors of early postpartum metabolic syndrome in Spanish mothers with gestational diabetes. Metabolic syndrome and related disorders;12(9):457 - 63.

5. Retnakaran R, Qi Y, Connelly PW, Sermer M, Zinman B, Hanley AJ. Glucose intolerance in pregnancy and postpartum risk of metabolic syndrome in young women. The Journal of Clinical Endocrinology Metabolism. 2010;95(2):670-7.

6. Malcolm J. (2012) Through the looking glass: gestational diabetes as a predictor of maternal and offspring long-term health. Diabetes/metabolism research and reviews;28(4):307 - 11. 
7. Baliutavičienè D, Buinauskienè JB, Petrenko V, Danytė E, Žalinkevičius R. (2012) Gestational diabetes, obesity, and metabolic syndrome diagnosed during pregnancy. Metabolic syndrome and related disorders;10(3):214-7.

8. Cho NH, Ahn CH, Moon JH, et al. (2016) Metabolic syndrome independently predicts future diabetes in women with a history of gestational diabetes mellitus. Medicine;95(35).

9. Lauenborg J, Mathiesen E, Hansen T, et al. The prevalence of the metabolic syndrome in a Danish population of women with previous gestational diabetes mellitus is three-fold higher than in the general population. The Journal of Clinical Endocrinology Metabolism. 2005;90(7):4004-10.

10. Shin JA, Lee JH, Lim SY, et al. (2013) Metabolic syndrome as a predictor of type 2 diabetes, and its clinical interpretations and usefulness. Journal of diabetes investigation;4(4):334 - 43.

11. Gunderson EP, Chiang V, Pletcher MJ, et al. History of gestational diabetes mellitus and future risk of atherosclerosis in mid-life: the coronary artery risk development in young adults study. Journal of the American Heart Association. 2014;3(2):e000490.

12. Alberti K, Eckel RH, Grundy SM, et al. Harmonizing the metabolic syndrome: a joint interim statement of the international diabetes federation task force on epidemiology and prevention; national heart, lung, and blood institute; American heart association; world heart federation; international atherosclerosis society; and international association for the study of obesity. Circulation. 2009;120(16):1640-5.

13. Ashrafi M, Gosili R, Hosseini R, Arabipoor A, Ahmadi J, Chehrazi M. Risk of gestational diabetes mellitus in patients undergoing assisted reproductive techniques. European Journal of Obstetrics Gynecology Reproductive Biology. 2014;176:149-52.

14. Kouhkan A, Khamseh ME, Pirjani R, et al. (2018) Obstetric and perinatal outcomes of singleton pregnancies conceived via assisted reproductive technology complicated by gestational diabetes mellitus: a prospective cohort study. BMC pregnancy and childbirth;18(1):495.

15. Kouhkan A, Khamseh ME, Moini A, et al. (2018) Predictive factors of gestational diabetes in pregnancies following assisted reproductive technology: a nested case-control study. Archives of gynecology and obstetrics:1-8.

16. Tobias DK, Chavarro JE, Williams MA, et al. (2013) History of infertility and risk of gestational diabetes mellitus: a prospective analysis of 40,773 pregnancies. American journal of epidemiology;178(8):1219-25.

17. Association AD. (2018) 2. Classification and diagnosis of diabetes: standards of medical care in diabetes-2018. Diabetes care;41(Supplement 1):S13-S27.

18. Grundy SM, Cleeman JI, Daniels SR, et al. (2005) Diagnosis and management of the metabolic syndrome: an American Heart Association/National Heart, Lung, and Blood Institute Scientific Statement. Circulation;112(17):2735-52.

19. Parikh RM, Mohan V. Changing definitions of metabolic syndrome. Indian journal of endocrinology metabolism. 2012;16(1):7. 
20. Xu Y, Shen S, Sun L, Yang H, Jin B, Cao X. (2014) Metabolic syndrome risk after gestational diabetes: a systematic review and meta-analysis. PloS one;9(1):e87863.

21. Nouhjah S, Shahbazian $\mathrm{H}$, Amoori N, et al. Postpartum screening practices, progression to abnormal glucose tolerance and its related risk factors in Asian women with a known history of gestational diabetes: A systematic review and meta-analysis. 11. Diabetes \& Metabolic Syndrome: Clinical Research \& Reviews; 2017. S703-S12.

22. Retnakaran R, Qi Y, Sermer M, Connelly PW, Hanley AJ, Zinman B. $\beta$-Cell function declines within the first year postpartum in women with recent glucose intolerance in pregnancy. Diabetes Care. 2010;33(8):1798-804.

23. Pallardo F, Herranz L, Garcia-Ingelmo T, et al. Early Postpartum Metabolic Assessment in Women With Prior Gestational Diabetes. Obstet Gynecol Surv. 2000;55(2):70.

24. Kramer CK, Swaminathan B, Hanley AJ, et al. (2014) Each degree of glucose intolerance in pregnancy predicts distinct trajectories of $\beta$-cell function, insulin sensitivity, and glycemia in the first 3 years postpartum. Diabetes care;37(12):3262-9.

25. Inoue H, Ishikawa K, Takeda K, et al. (2018) Postpartum risk of diabetes and predictive factors for glucose intolerance in East Asian women with gestational diabetes. Diabetes research and clinical practice;140:1-8.

26. Wahabi H, Fayed A, Tunkar SM, et al. (2019) Incidence and contributing factors of glucose intolerance in Saudi postpartum women: Sub-group analysis from RAHMA study. PloS one;14(1):e0210024.

27. Youngwanichsetha S, Phumdoung S. Factors related to prediabetes among postpartum Thai women with a history of gestational diabetes mellitus. Nurs Health Sci. 2013;15(4):449-53.

28. Coetzee A, Mason D, Hall DR, Conradie M. Prevalence and predictive factors of early postpartum diabetes among women with gestational diabetes in a single-center cohort. International Journal of Gynecology Obstetrics. 2018;142(1):54-60.

29. Nouhjah S, Shahbazian H, Shahbazian N, Jahanshahi A, Jahanfar S, Cheraghian B. (2017) Incidence and contributing factors of persistent hyperglycemia at 6-12 weeks postpartum in Iranian women with gestational diabetes: results from LAGA Cohort Study. Journal of diabetes research;2017.

30. Guo X-Y, Liu X-M, Jin L, et al. Cardiovascular and metabolic profiles of offspring conceived by assisted reproductive technologies: a systematic review and meta-analysis. Fertility sterility. 2017;107(3):622-31. e5.

31. Catford S, McLachlan R, O'Bryan M, Halliday J. (2018) Long-term follow-up of ICSI-conceived offspring compared with spontaneously conceived offspring: a systematic review of health outcomes beyond the neonatal period. Andrology;6(5):635 - 53.

32. Pontesilli M, Painter RC, Grooten IJ, et al. Subfertility and assisted reproduction techniques are associated with poorer cardiometabolic profiles in childhood. Reprod Biomed Online. 2015;30(3):258-67. 
33. Vannuccini S, Clifton VL, Fraser IS, et al. Infertility and reproductive disorders: impact of hormonal and inflammatory mechanisms on pregnancy outcome. Hum Reprod Update. 2016;22(1):104-15.

34. Chen M, Wu L, Zhao J, et al. (2014) Altered glucose metabolism in mouse and humans conceived by IVF. Diabetes;63(10):3189-98. 